\title{
Intraductal Papillary Mucinous Neoplasms of the Pancreas: Strategic Considerations
}

\author{
Vicente Morales-Oyarvide Zhi Ven Fong Carlos Fernández-del Castillo Andrew L. Warshaw
}

Department of Surgery, Massachusetts General Hospital and Harvard Medical School, Boston, MA, USA

\section{Keywords}

Intraductal papillary mucinous neoplasm, IPMN .

Pancreatic cysts · Management

\section{Summary}

Intraductal papillary mucinous neoplasms (IPMN) are cystic neoplasms with the potential for progression to pancreatic cancer. Recognized by the global medical community just over two decades ago, IPMN have gained great epidemiological and clinical relevance thanks to the widespread use of cross-sectional abdominal imaging, which has led to a surge in the number of incidental pancreatic cysts being diagnosed. As our understanding of this disease has improved, we now know that some IPMN have a very elevated risk of cancer and require surgical resection, while others are low-risk lesions and can be followed. The approach to IPMN must therefore strike a balance between preventing the overutilization of surgery and the timely recognition and treatment of patients with high-risk lesions. Several clinical, radiographic, and laboratory parameters have been proposed to risk-stratify IPMN, leading to the publication of management guidelines that do not always converge in their recommendations. The goal of this clinical therapeutic review is to describe the strategic approach to IPMN at the Massachusetts General Hospital, and how our current understanding, management algorithm, and future directions have been informed by research efforts at our institution and other centers.

(C) 2017 S. Karger GmbH, Freiburg

\section{Introduction}

Intraductal papillary mucinous neoplasms (IPMN) have transformed the field of pancreatology. Regardless of the lens through which one approaches the study of the pancreas, the field as we know it today would be unrecognizable without IPMN and other pancreatic cystic neoplasms. Virtually unknown by the international medical community 25 years ago, IPMN account for nearly half of pancreatic resections for cystic lesions at the Massachusetts General Hospital (MGH) between 2005 and 2011 [1]. Our understanding of this disease has evolved dramatically over the past few decades. IPMN demonstrate a spectrum of cytomorphological atypia that is defined as low-grade dysplasia (LGD), high-grade dysplasia (HGD), and invasive carcinoma [2]. It is now clear that not all IPMN are the same: some affect the main pancreatic duct (MPD) extensively, have different clinical presentations, and pose a high risk of harboring an invasive carcinoma; while others remain confined to the side branches of the ductal system, are asymptomatic, and are considered low-risk lesions. Further, it is becoming ever more clear that invasive carcinomas arising from IPMN are distinct from 'conventional' pancreatic ductal adenocarcinomas (PDAC), demonstrating important morphological and genetic differences that have major prognostic implications [3-7].

The management of IPMN is complex; not too long ago, treatment approaches were based predominantly on institutional and individual experience. In 2004, a group of experts convened in Sendai, Japan, and drafted the first international consensus guidelines for the management of IPMN and mucinous cystic neoplasms $(\mathrm{MCN})$ of the pancreas under the auspices of the International Association of Pancreatology (IAP) [8]. The IAP Sendai consensus guidelines (as they became known) were the first international effort to standardize treatment and follow-up for patients with this condition. A few years later and after several validation studies, these guidelines were updated following a meeting in Fukuoka, Japan, and the IAP 2012 Fukuoka consensus guidelines became the 
Fig. 1. Intraductal papillary mucinous neoplasm (IPMN) types based on main pancreatic duct (MPD) involvement. A Main duct IPMN; contrastenhanced computed tomography showing a heterogeneous, hypodense lesion in the
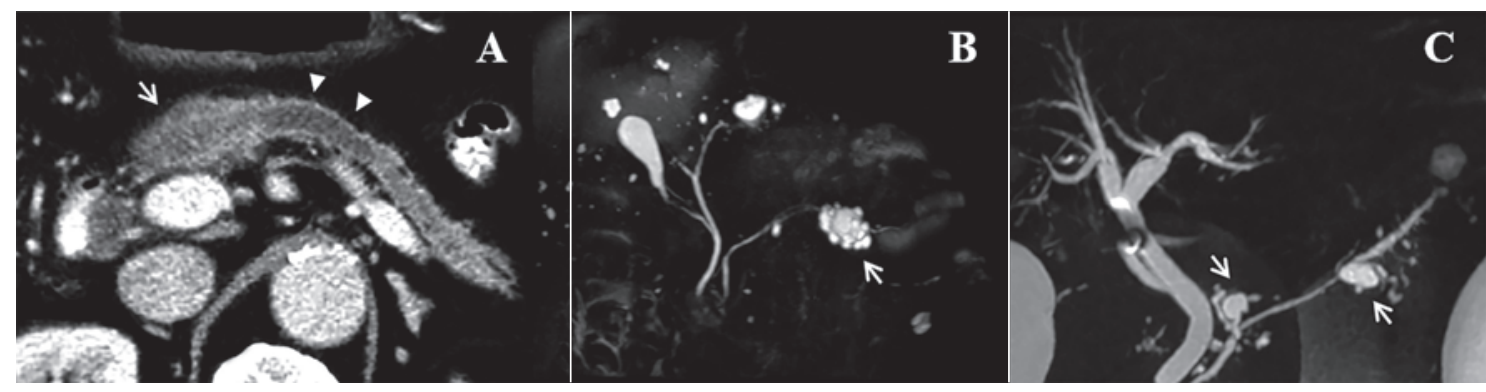

pancreatic neck (arrow) with dilation of the MPD (arrow heads) and distal atrophy. B Branch duct IPMN; magnetic resonance with cholangiopancreatography (MRCP) demonstrating a T2 hyperintense lesion (arrow) in the pancreatic body showing communication with the MPD. C Mixed-type IPMN; MRCP revealing cystic lesions in the head and body of the pancreas, communicating with the MPD (arrows); involvement of the MPD is not apparent on radiographic imaging but was found on histological examination after resection (mixed-type IPMN with minimal histological involvement of the MPD).

preponderant set of recommendations [9]. The safety and efficacy of these guidelines has been assessed extensively, and they were further revised in 2017, with only minor modifications [10].

With increased awareness and widespread use of cross-sectional abdominal imaging, the number of patients diagnosed with pancreatic cysts has only continued to grow and it has become a preeminent issue for the gastroenterological community. As a result, in 2015, the American Gastroenterological Association (AGA) put forth the AGA Institute guidelines on the diagnosis and management of pancreatic cysts [11] following an exhaustive technical review of the literature [12]. As we will discuss later in this article, the IAP and AGA guidelines exhibit critical contrasts that reflect our still limited understanding of the natural history of IPMN and how much more we must learn. At MGH, we have strived to be at the cutting edge of the research efforts to better understand the biology and behavior of IPMN, and while we have mostly followed the IAP guidelines in our clinical practice, our perspective may not always align with every aspect of the latest international consensus. Our goal in this article is to provide an overview of the MGH institutional experience with IPMN and of how our research - often in collaboration with other centers - has helped to inform our strategic approach to these tumors.

\section{Epidemiology of Intraductal Papillary Mucinous Neoplasms}

The true prevalence of IPMN is not known, but the generalized use of cross-sectional imaging has allowed us to conclude that pancreatic cysts become common with increasing age [13, 14]. Calculating the prevalence of incidental (i.e. asymptomatic) pancreatic cysts in the general population is a complex task, as most studies have included patients from tertiary care centers who may have a history of liver or pancreatobiliary conditions, thus introducing potential for referral bias $[14,15]$. To overcome this limitation, a study by de Jong et al. [16] assessed the prevalence of pancreatic cysts in 2,803 consecutive subjects (mean age 51.1 years) who underwent screening with abdominal magnetic resonance imaging (MRI) as part of a preventive medical examination. The authors identified pancreatic cysts in $2.4 \%$ of the subjects, and their pres- ence was strongly correlated with age: less than $1 \%$ of subjects between 18 and 49 years of age had a pancreatic cyst, compared to $10 \%$ of individuals older than 70 years. Importantly, most cysts were smaller than $1 \mathrm{~cm}$ (median size $8 \mathrm{~mm}$ ), with only a few being larger than $2 \mathrm{~cm}$; among patients with pancreatic cysts, $12 \%$ were multifocal. There is no robust evidence on the exact pathological diagnosis of these asymptomatic pancreatic cysts, but it is believed that a large proportion of them are small IPMN confined to the ductal side branches. While IPMN have traditionally been considered more common in men, especially in surgical series, there was no gender difference in the observed prevalence of pancreatic cysts. Our institutional experience is consistent with the above findings in relation to patient age and sex: the median age at initial diagnosis of 577 patients with suspected or presumed IPMN undergoing surveillance at MGH was 66 years, and 59\% were women [17].

Types of Intraductal Papillary Mucinous Neoplasms Based on Main Pancreatic Duct Involvement:

\section{The First Layer of Classification}

IPMN are primarily classified as main duct (MD-IPMN), branch duct (BD-IPMN), or mixed-type IPMN based on the extent to which they involve the pancreatic ductal system (fig. 1). Preoperatively, this distinction is defined by the diameter of the MPD on imaging studies: according to the revised IAP 2012 Fukuoka consensus guidelines [10], segmental or diffuse dilation of the MPD of $>5 \mathrm{~mm}$ without other causes of obstruction defines MD-IPMN (fig. 1A); pancreatic cysts $>5 \mathrm{~mm}$ in size demonstrating communication with the MPD but with no MPD dilation are classified as BD-IPMN (fig. 1B); and lesions meeting criteria for both MD- and BD-IPMN are classified as mixed-type IPMN. This classification is of biological and practical importance: based on a summary of 20 different studies including 3,568 IPMN, the risk of invasive carcinoma arising in association with MD-IPMN is approximately $44 \%$, compared to approximately $17 \%$ in BD-IPMN [9]. These estimates vary widely across centers, in part because some studies classify tumors based on their degree of histological - rather than radiological - MPD involvement, and the two criteria have shown a modest correlation (approximately 70\%) [18, 19]. In our institution, the 
Fig. 2. The precursor and invasive components of intraductal papillary mucinous neoplasms (IPMN) demonstrate morphological and genetic heterogeneity. Based on its cytoarchitectural features, the precursor component is classified as gastric, pancreatobiliary, oncocytic, or intestinal. Invasive carcinomas arising from gastric- and pancreatobiliary-type IPMN exhibit a tubular/ductal morphology, similar to that of conventional pancreatic ductal adenocarcinoma; in comparison, most invasive carcinomas arising from intestinal-type IPMN are of the colloid type. Oncocytic-type IPMN can give rise to invasive carcinoma, either superficially invasive, of tubular type, or of oncocytic type.

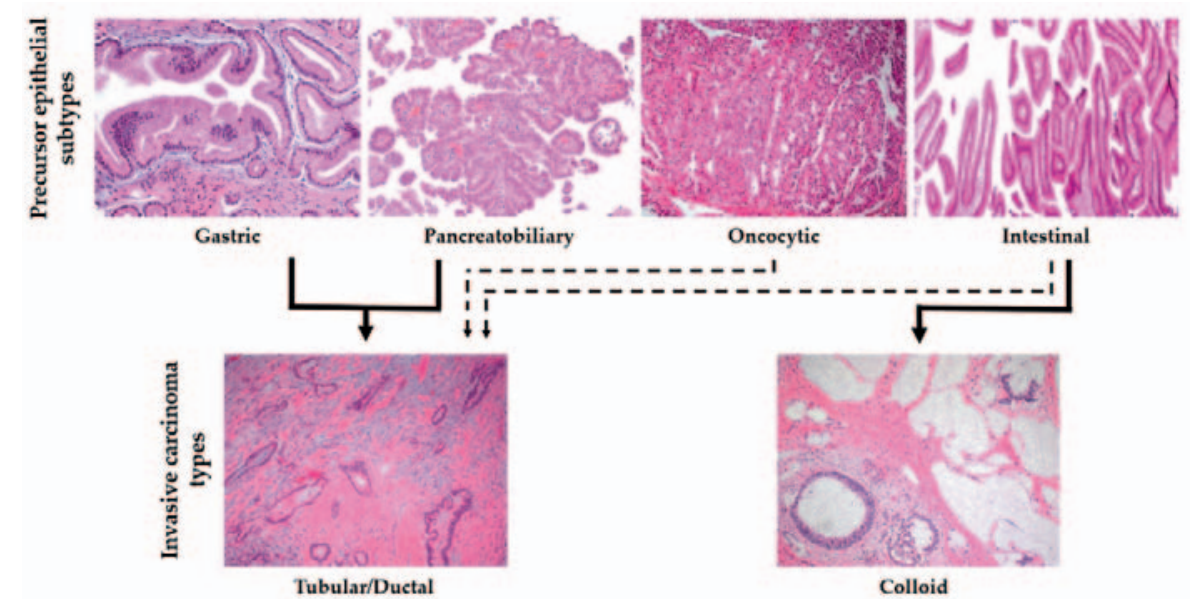

risk of HGD and invasive carcinoma in resected, radiologically defined MD-IPMN is 32 and $47 \%$, respectively; in comparison, the risk of HGD and invasive carcinoma in resected BD-IPMN is 15 and $9 \%$. We must emphasize that these risk estimates derive from resected IPMN; since most BD-IPMN are not resected, their actual risk of HGD and invasive carcinoma must be much lower. Naturally, these differences in risk between $\mathrm{MD}$ - and BD-IPMN have a huge influence on the treatment strategy for each IPMN type: based on the revised IAP 2012 Fukuoka consensus guidelines [10], radiological MPD involvement is an indication for resection in surgically fit patients; in comparison, patients with BD-IPMN may be offered surgery or surveillance based on the presence of clinical and radiological worrisome features and high-risk stigmata, as we will detail later in this article.

Mixed-type IPMN, on the other hand, have been a source of growing interest in recent years. They have historically been considered akin to MD-IPMN (the revised 2012 Fukuoka consensus guidelines recommend identical management for both types), but evidence suggests that not all mixed-type IPMN are equal. At MGH, mixed-type IPMN defined by current imaging criteria have a risk of HGD and invasive carcinoma of 31 and 28\%, respectively: higher than that of BD-IPMN, but lower than MD-IPMN. Detailed histological analysis of these tumors by our group has shed light on this 'mixed' behavior [20]. Pathological re-review of 404 resected IPMN revealed that some mixed-type IPMN (approximately 1 of every 5) demonstrate only minimal histological involvement of the MPD (non-circumferential microscopic involvement), and this subgroup had a risk of HGD (11\%) and invasive carcinoma (6\%) comparable to that of histologically-defined BD-IPMN (fig. 1C); in contrast, mixed-type IPMN with extensive MPD involvement had a significantly higher risk of HGD (37\%) and invasive carcinoma (33\%). These data suggest that mixed-type IPMN with minimal histological MPD involvement may have a more indolent behavior and could perhaps be managed in a similar way as BD-IPMN. At this point, the distinction between mixed-type IPMN with minimal versus extensive histological MPD involvement can only be made after surgery, when it is only of little relevance compared with the identification of invasive carcinoma, but preliminary data from our institution indicate that preoperative recognition of histological
MPD involvement may be feasible. We found that MPD diameter as measured by computed tomography (CT) or MRI is effective at distinguishing extensive versus minimal histological MPD involvement (area under the curve (AUC) 0.85; 95\% confidence interval (CI) $0.80-0.90)[21]$.

\section{Epithelial Subtypes of the Precursor Component and the Invasive Carcinoma: Insights into Tumor Biology and Its Heterogeneity}

IPMN are characterized by papillary formations that project into the pancreatic ductal system. The epithelial lining of these papillae displays significant morphologic heterogeneity that has led to the recognition of four distinct epithelial subtypes of the precursor component (i.e. non-invasive component), namely: gastric, intestinal, pancreatobiliary, and oncocytic (fig. 2) [22, 23]. Since it is not rare to encounter more than one epithelial subtype in each tumor, IPMN are classified based on the predominant subtype. Invasive carcinomas arising from IPMN also exhibit important histological differences and are classified as tubular (ductal), colloid, and oncocytic types (fig. 2) [22, 23]. The recognition of different precursor and invasive phenotypes has important prognostic implications, and it is becoming increasingly clear that these subgroups may represent specific pathways of differentiation with distinct molecular features [5, 6, 24]. Gastric-type epithelium is most common in BD-IPMN and is often associated with LGD (77\% of resected gastric-type IPMN at our institution); paradoxically, when invasive carcinoma arises from a gastric-type IPMN, it is usually of tubular type, which carries a poor prognosis akin to that of conventional PDAC [3]. As in PDAC, KRAS mutations are highly prevalent in gastric-type IPMN and tubular-type carcinomas [25, 26]. In contrast, intestinal-type epithelium is predominantly found in MD-IPMN, is associated with HGD and invasive carcinoma (40 and $33 \%$ of intestinal-type IPMN at our institution, respectively), and often gives rise to colloid-type carcinoma, which has more favorable clinicopathological features and prognosis [3]. Moreover, intestinal-type IPMN and colloid-type carcinomas exhibit GNAS mutations and markers of intestinal differentiation (e.g. MUC2 
positivity and CDX2 expression) [24-26]. Pancreatobiliary-type epithelium is uncommon ( $<5 \%$ of IPMN resections at MGH), and in many cases it is hard to distinguish from gastric-type IPMN with HGD [23]; it virtually always demonstrates HGD or invasive carcinoma (47 and $53 \%$ at our institution, respectively), and when it is invasive it gives rise to tubular-type carcinomas. Of the four epithelial subtypes, oncocytic-type IPMN is perhaps the most unique. Highly infrequent (accounting for only $4 \%$ of resections at MGH), this subtype is associated with MPD involvement, HGD, and invasive carcinoma of the oncocytic type, but has surprisingly favorable outcomes: in a series of 18 patients from our hospital, the median overall survival (OS) after resection was more than 10 years and there were no disease-specific deaths [27]. Given the above considerations, the revised IAP 2012 Fukuoka consensus guidelines emphasize the importance of classifying invasive carcinomas arising from IPMN as tubular, colloid, or oncocytic [10], and this is our standard practice. Whether the different types of invasive carcinoma exhibit distinct response to adjuvant chemotherapy and radiation remains unclear, and we need more research on this topic to continue tailoring patient treatment and follow-up.

We recently reported that age plays a role in IPMN heterogeneity [28]. In a series of 1,693 resected IPMN from the institutions conforming the Pancreatic Surgery Consortium (MGH, Memorial Sloan Kettering Cancer Center, and the Johns Hopkins Hospital), we found that IPMN from patients who were younger than 50 years of age at the time of resection were more likely to be of the oncocytic and intestinal subtypes; moreover, they were less likely to harbor an invasive carcinoma, and when this was present, it was more often of colloid type and associated with improved OS. We continued to observe these associations - albeit in lesser magnitude - using age cutoffs of 55 and 60 years of age. Notably, only $5 \%$ of patients undergoing resection were younger than 50 , a fact that underscores the tremendous importance of multi-center collaborations if we are to characterize uncommon subsets of patients that would otherwise be too rare in single-center series.

\section{Clinical Presentation}

We will next discuss the different information and tools available for identifying patients with high-risk lesions. Most IPMN currently being diagnosed are asymptomatic (i.e. incidental), especially BD-IPMN. In a series of 563 BD-IPMN (411 primarily observed and 152 primarily resected) from our institution, 57\% were asymptomatic [29]. In a follow-up study limited to patients with a minimum of 6 months of surveillance (i.e. excluding those who were offered surgery soon after diagnosis), $83 \%$ were asymptomatic at initial diagnosis, and of these, only $10 \%$ went on to become symptomatic during follow-up [17]. As one would expect, the rate of symptomatic IPMN is higher in surgical series. Half of patients who have undergone resection for IPMN at MGH were symptomatic, with the most common symptom being abdominal pain (41\%), followed by weight loss $>10$ lbs (29\%), acute pancreatitis (22\%), and jaundice (9\%).

Intraductal Papillary Mucinous Neoplasms of the Pancreas
Diabetes mellitus - especially of recent onset - is a common finding in patients with conventional PDAC [30], but its role as a harbinger of HGD and invasive carcinoma in IPMN has not been well studied. We recently reported that diabetes mellitus is common among patients undergoing resection for IPMN (34\%), and it is associated with a twofold elevated risk of HGD and invasive carcinoma (analyzed as separate endpoints) after adjusting for IAP 2012 Fukuoka worrisome features [31]. Moreover, recent-onset diabetes mellitus (diagnosed $\leq 5$ years before surgery) was associated with 6.9-fold increased risk of invasive carcinoma ( $<<0.001)$. If further studies validate the independent role of diabetes mellitus in the prediction of HGD and invasive carcinoma in IPMN, it could be considered an additional element of the management algorithm.

In addition to being associated with degree of dysplasia, some symptoms of presentation may be informative of the IPMN epithelial subtypes. We have reported that IPMN from patients presenting with acute pancreatitis are 4.8 times more likely to be of the intestinal subtype [32]. Similarly, a diagnosis of diabetes mellitus is associated with 1.6-fold and 2.5-fold higher likelihood of intestinal-type IPMN and colloid-type carcinoma, respectively [31]. As we discussed previously, intestinal-type IPMN usually involve the MPD and secrete MUC2-rich, viscous mucin. We hypothesize that these thick secretions may lead to duct obstruction and pancreatic inflammation, which could be associated with episodes of acute pancreatitis and perhaps with development of diabetes mellitus, but at this point this is merely speculative.

\section{Circulating Biomarkers for Prediction of High-Risk Intraductal Papillary Mucinous Neoplasms}

Multiple circulating biomarkers have been assessed as potential indicators of HGD or invasive carcinoma in IPMN. Of those, the most widely studied is the carbohydrate antigen 19-9 (CA 19-9), an independent predictor of disease stage and survival in patients with resectable, conventional PDAC [33]. In a recent meta-analysis of fifteen studies and a total of 1,629 patients [34], elevated serum CA 19-9 had a pooled sensitivity and specificity of 52 and $88 \%$ for detecting invasive carcinoma in IPMN, respectively. Unfortunately, the authors did not conduct analyses using HGD without invasive carcinoma as a separate study endpoint. Data from our institution [35] indicate that serum CA 19-9 in patients with resected BDIPMN is useful for detecting invasive carcinoma (AUC 0.68; 95\% CI 0.52-0.84) but not for detecting HGD without invasive carcinoma (AUC 0.54; 95\% CI 0.42-0.67). Importantly, a cutoff of 100 units $/ \mathrm{ml}$ had the highest accuracy for detecting invasive carcinoma (93\%), compared with the more commonly used cutoff of 37 units/ $\mathrm{ml}(83 \%)$ [36, 37]. Elevated serum CA 19-9 has now been included as a worrisome feature in the revised IAP 2012 Fukuoka consensus guidelines [10]; while our experience would support this addition, we must keep in mind that its utility appears to be restricted to detecting invasive carcinoma (and not HGD), and the optimal cutoff value may still need to be determined. 
Fig. 3. Endoscopic and ultrasonographic evaluation of intraductal papillary mucinous neoplasms (IPMN). A Upper endoscopy demonstrating a patulous, 'fish-mouth' papilla extruding mucus, pathognomonic of IPMN affecting the main pancreatic duct. B Endoscopic ultrasound revealing a mural nodule within the cyst, a known harbinger of high-grade dysplasia and invasive carcinoma in IPMN.

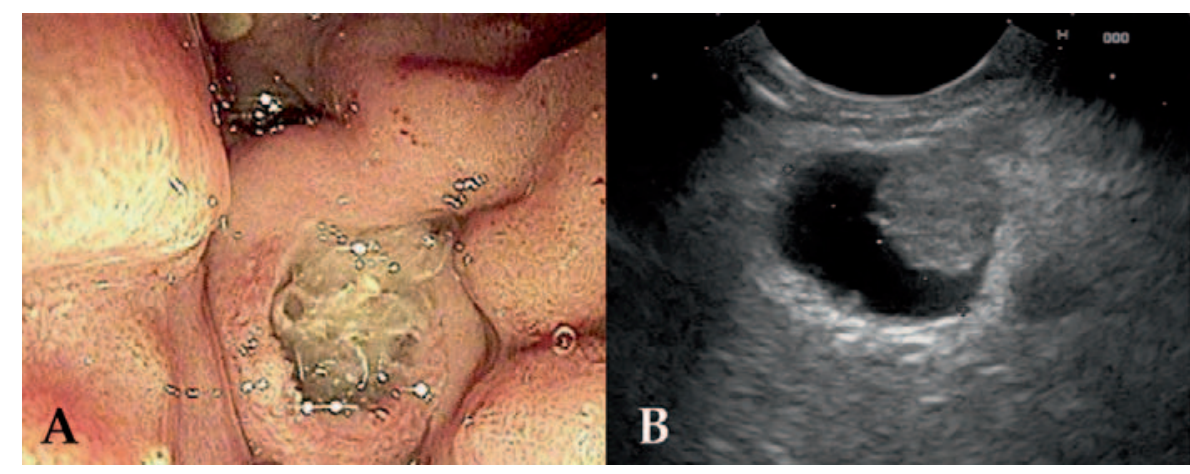

More recently, the neutrophil-to-lymphocyte ratio (NLR) in blood has been evaluated as a novel predictor of HGD and invasive carcinoma, which could presumably reflect the systemic immune response elicited by cancer, among other potential physiologic mechanisms $[38,39]$. In a series of 272 patients with resected IPMN from the Johns Hopkins Hospital, Gemenetzis et al. [39] found that an NLR > 4 was significantly associated with invasive carcinoma, independent of MPD size, presence of mural nodules, jaundice, and cyst size. Notably, a higher NLR was not associated with HGD without invasive carcinoma. At our institution, we do not systematically use blood NLR in the assessment of patients with IPMN, but we recognize its practical potential as a biomarker and the need for validation studies. Similar to serum CA 19-9, its main shortcoming lies in the limited ability to detect HGD without invasive carcinoma.

\section{Imaging Evaluation}

Different cross-sectional and ultrasonographic imaging modalities are used in the initial assessment and surveillance of patients with IPMN, each with its own strengths and limitations. Imaging studies pursue two primary goals: to distinguish IPMN from other pancreatic cysts, and to identify MPD involvement to determine IPMN with higher risk of harboring HGD or invasive carcinoma. Multidetector CT (MDCT) and MRI with cholangiopancreatography (MRCP) are effective and reliable methods to characterize cyst morphology, size, and communication with the MPD. In our experience, MDCT and MRCP can distinguish mucinous versus non-mucinous cysts with an accuracy of $71-85 \%$ $[40,41]$. The risk assessment of lesions suspected to be IPMN is centered on the recognition of MPD involvement and presence of mural nodules within or adjacent to the cyst, both known markers of HGD and invasive carcinoma. Accurately identifying mural nodules has proven to be a challenge, since they are often hard to distinguish from mucin plugs. In an effort to further understand the evaluation of mural nodules, studies have shown that nodule size can help identify patients at higher risk of HGD and invasive carcinoma [42, 43]. In the original IAP 2012 Fukuoka consensus guidelines, presence of an enhancing mural nodule was defined as a high-risk stigma without further consideration of nodule size; the new revised IAP 2012 Fukuoka consensus guidelines recom- mend that mural nodules $\geq 5 \mathrm{~mm}$ be considered a high-risk stigma, while a size smaller than $5 \mathrm{~mm}$ be regarded as a worrisome feature [10]. More research is needed to determine whether we should be concerned about the size of mural nodules, and if so, what the optimal size threshold is.

Our experience and that of other centers show that both MDCT (especially dual-phase pancreatic protocol CT) and gadolinium-enhanced MRI are adequate for initial evaluation and surveillance of suspected IPMN, with an accuracy to identify lesions with HGD and invasive carcinoma in the range of $75-86 \%$ in two contemporary studies from our institution [40,41,44]. While both imaging modalities have demonstrated comparable diagnostic performance [40, 44], MRI is favored as the method of choice for surveillance of suspected IPMN [45]. This is partly because MRI may allow for superior visualization of the ductal system anatomy, presence of internal septations, and mural nodules, but more importantly because it spares patients under long-term surveillance from repeated radiation exposure that would otherwise occur with MDCT.

Various endoscopic modalities are used to further characterize suspected IPMN. Upper endoscopy can reveal the classic, patulous 'fish mouth' papilla extruding mucus, which is pathognomonic of IPMN involving the MPD (fig. 3A) [46, 47], although the trend towards early diagnosis of small, incidental lesions has made this finding increasingly rare. Endoscopic retrograde cholangiopancreatography with or without pancreatoscopy has been used to obtain pancreatic juice and brushings for cytology, and to visualize papillary or villous growths along the ducts, but evidence to support its routine use is lacking and we do not recommend it [10, 47]. Endoscopic ultrasound (EUS) has become a highly valuable tool in the diagnostic work-up of IPMN; guidelines make different recommendations regarding who should be investigated with this technique, as we will discuss in a later section. One of the advantages of EUS is its superior ability to detect mural nodules, one of the stronger predictors of high-risk IPMN (fig. 3B), albeit with risk of false positives due to the mucin plugs mentioned earlier [48-50]. This caveat can be avoided using contrast-enhanced harmonic EUS, which allows the operator to reliably detect blood flow in the suspected nodule and thus to better distinguish mucin plugs from true mural nodules with a sensitivity and specificity of $89-96 \%$ and 64-88\%, respectively [51]. Beyond the detailed characterization of IPMN morphology, EUS offers another diagnostic advantage: it al- 
lows us to perform EUS-guided fine-needle aspiration of the cyst contents, paving the way for comprehensive cyst fluid analysis and cytology.

\section{Cyst Fluid Analysis: Proteins, Cytology, and Molecular Characterization}

Our understanding of pancreatic cyst fluid analysis continues to evolve and, for the most part, remains investigational. For the purposes of this discussion, we will classify the different cyst fluid parameters of interest as cyst fluid protein (antigen) analysis, cytological analysis, and molecular analysis. Carcinoembryonic antigen (CEA) is the most widely studied protein marker in pancreatic cyst fluid. It cannot distinguish between benign cysts and those with HGD or invasive carcinoma [52], but it is very valuable to distinguish mucinous from non-mucinous lesions. A cooperative study between MGH and several other centers found in 2004 that a CEA cutoff value of $192 \mathrm{ng} / \mathrm{ml}$ had the greatest AUC (0.79; accuracy 79\%) to detect mucinous cysts [53]. A more recent study including nearly twice as many patients from our institution showed that a cutoff value of $109.9 \mathrm{ng} / \mathrm{ml}$ is more accurate (86\%) and has a greater AUC (0.93) [54]. Neither the IAP nor the AGA guidelines set cyst fluid CEA thresholds in their recommendations, but 192 $\mathrm{ng} / \mathrm{ml}$ remains the most consistently used value.

In a collaborative effort with the Rutgers Robert Wood Johnson Medical School, we have also found that mAb Das-1, a monoclonal antibody that reacts specifically with normal colonic epithelium and intestinal metaplasia of the lower esophagus (Barrett's esophagus), can identify high-risk IPMN using cyst fluid with a sensitivity and specificity of 89 and $100 \%$, respectively [55]. A preliminary, multi-institutional validation study of the performance of $\mathrm{mAb}$ Das-1 confirmed our initial findings and suggests that this antibody may be a valuable addition to current clinical guidelines [56].

The interpretation of cyst fluid cytological and molecular analyses presents a greater challenge. Even in referral centers with highly specialized endoscopists and cytopathologists, the sensitivity of pancreatic cyst fluid cytology is low due to insufficient aspirated volume, scant cellularity, and contamination with gastric and duodenal mucosal cells. These hurdles make it difficult to reach a formal determination of 'positive' cytology. Cytopathologists from our institution have shown that lowering the threshold from 'positive' cytology to presence of high-grade atypical epithelial cells (which are quantitatively and qualitatively insufficient for a positive cytology interpretation), we can achieve an increase in the accuracy (80-85\%) and in the number of cases of HGD and invasive carcinoma detected (12\% more than when using 'positive' cytology) $[57,58]$. Lastly, the continuous progress in genomic technologies holds the promise of accurate diagnosis and risk stratification of pancreatic cysts using molecular analysis of cyst fluid. Most studies have focused on determining alterations in KRAS and GNAS, two of the more common gene alterations in IPMN; the former are considered highly specific of mucinous lesions (also occurring in some $\mathrm{MCN}$ ) and are found in the fluid of approximately half of IPMN, while the latter are believed to be specific of IPMN (especially so in those demonstrating intestinal-colloid differentiation) and are observed in the cyst fluid of approximately two thirds of IPMN (mutations in either gene are detected in $45-96 \%$ of IPMN) $[5,26,59]$. Unfortunately, regardless of how sensitive or specific mutations in these genes may be for differentiating IPMN from other cystic lesions, they do not inform risk of HGD or invasive carcinoma. Recent efforts from our center and others have shown that performing next-generation sequencing in fluid from pancreatic cysts is feasible [60], allowing for the identification of mutations in several genes whose exact role in diagnosis and management is still undetermined but may prove very useful in the near future.

Ultimately, the information we obtain from protein, cytological, and molecular analyses of cyst fluid will be part of a larger mosaic that incorporates a patient's clinical picture, serum markers, and imaging findings. Indeed, a recent multi-center study from several countries offered promising results using a combination of clinical features and molecular markers, allowing for determination of pancreatic cyst type with a sensitivity of $90-100 \%$ and specificity of 92-98\% [61].

\section{Considerations Regarding Operative Approach to Intraductal Papillary Mucinous Neoplasms}

Patients with completely resected non-invasive (i.e. LGD or HGD) IPMN have excellent outcomes, with >95\% 10-year diseasespecific survival [62]. In contrast, patients with resected invasive carcinoma have a poor prognosis, with 5- and 10-year OS rates of 47 and 34\%, respectively, in our experience [3]. Among these, outcomes differ based on the type of invasive carcinoma: tubular-type carcinoma is associated with 5 -year OS rates of $37 \%$, whereas 5 -year OS for colloid carcinoma is approximately 61\% [3]. These survival estimates clearly demonstrate that IPMN need to be diagnosed and resected at an early stage; they also show that the distinction between HGD and invasive carcinoma is of paramount importance. This distinction is not clear in many studies, limiting our ability to compare different series and putting two groups of patients with wide contrasts in one basket. While we are not certain about the natural history of IPMN with HGD, waiting for invasive carcinoma to develop in a disease as lethal as pancreatic cancer would render our surveillance efforts pointless. We therefore strongly recommend that studies evaluating potential new markers for risk stratification of IPMN analyze HGD and invasive carcinoma as two separate endpoints, with a strong emphasis on HGD.

Mortality associated with pancreatic resection has decreased substantially. In the last two decades, surgical-related death after a pancreatoduodenectomy at MGH occurred in $1.5 \%$ of patients, with septic shock being the most common cause of death (33\%) [63]. Surgical morbidity, however, remains an issue. Up to $45 \%$ of patients undergoing surgery for IPMN suffer from a complication (e.g. delayed gastric emptying, wound infection, cardiopulmonary complications), with $12 \%$ of complications being pancreatic fistu- 
las. Despite the high morbidity rate, patients undergoing pancreatoduodenectomy eventually report high health-related quality of life and functional status 5 years after the procedure [64].

The presence of comorbidities is an important consideration when deciding in which patients to recommend pancreatic resection. Patients with a high comorbidity burden who are at high risk of non-IPMN-related death may derive little to no benefit from active surveillance or pancreatic surgery. Using the age-adjusted Charlson comorbidity index (CACI), we found that in a group of 725 patients who underwent resection or were under surveillance for IPMN at MGH, the median CACI score was 3 [65]. 10\% of patients had a $\mathrm{CACI} \geq 7$ (indicating severe comorbidities), and their median OS was 43 months. Compared to patients with a CACI $<7$, those with a CACI $\geq 7$ had an 11-fold higher risk of dying from non-IPMN-related causes within 3 years. Thus, patients with severe comorbidity burden are at greater risk of non-IPMN-related death, even in the presence of invasive carcinoma. Based on these findings, neither active surveillance nor resection would be justified in this subgroup.

The standard surgical approach for patients with IPMN meeting indications for resection is pancreatoduodenectomy, left (distal) pancreatectomy, or total pancreatectomy depending on the location and extension of the tumor, all accompanied by lymph node dissection [10]. A growing number of studies has recently advocated for increased consideration of limited, non-anatomic resections (e.g. enucleation, middle pancreatectomy) for some instances of BD-IPMN. These parenchymal-sparing approaches may manage to preserve pancreatic exocrine and endocrine function, albeit sometimes with high rates of short-term surgical morbidity [6668]. From our perspective, the idea of performing enucleation of a tumor communicating with the MPD is problematic (i.e., by definition, all IPMN). If it is felt that an IPMN poses an elevated risk of HGD or invasive carcinoma and needs to be resected, then enucleation would not be a sound approach from an oncologic standpoint. Additionally, if the risk of HGD or invasive carcinoma is thought to be so low that enucleation would be an appropriate procedure, that patient should not have undergone any pancreatic resection in the first place. Considerations about the 'radicality' of surgery (i.e. resection margin status, IPMN left behind in the remnant pancreas) will be discussed in the next section in the context of follow-up after resection and prediction of disease recurrence.

\section{Follow-up of Resected Patients: Predicting Recurrent and New Lesions}

Disease recurrence remains a concern after primary resection for IPMN. We define recurrence as presence of new IPMN on imaging studies, either i) at the resection margin of the primary surgery or ii) in the pancreas remnant, peripancreatic lymph nodes, or distant organs. Known ('residual') IPMN located away from the resection margin and purposefully left behind during primary surgery are not recurrent lesions and should be assessed individually. As in most other matters involved in the management and out- comes of patients with IPMN, the risk of recurrence depends heavily on the degree of MPD involvement and on the presence of invasive carcinoma in the resected tumor. In our experience, the overall recurrence rate for resected IPMN is around 17\%, and of those patients, around $14 \%$ require a second surgery [69]. The relatively low rate of reoperation in patients who recur is because not all recurrences are equal: some IPMN recur in the form of an invasive carcinoma, while others do so as non-invasive lesions. Nearly half of patients undergoing resection for IPMN with invasive carcinoma experience recurrence within a median of 19 months, which is virtually always invasive. These recurrences occur more commonly in patients with lymph node metastases and in those with tubular-type carcinomas. Conversely, less than $10 \%$ of non-invasive IPMN recur, and in the case of non-invasive BD-IPMN, the recurrence is almost always a non-invasive lesion and occurs after a median of more than 4 years after primary resection. Consequently, and in accordance with the revised IAP 2012 Fukuoka consensus guidelines, we recommend that patients undergoing resection for IPMN with invasive carcinoma should continue surveillance similar to that of conventional PDAC [10].

The follow-up of patients with resected, non-invasive IPMN is more controversial. Our experience shows that approximately $9 \%$ of non-invasive IPMN recur, consistent with other referral centers that have reported recurrence rates of $5-8 \%$ in this patient subgroup [70, 71]. Furthermore, it is becoming clearer that IPMN are associated with a 'field defect', whereby the entire pancreas would be affected and at higher risk of pancreatic neoplasia, a hypothesis driven in part by the occurrence of synchronous, multifocal disease and concomitant (concurrent) PDAC arising separately from IPMN. In our experience, up to $47 \%$ of BD-IPMN are multifocal and the number of lesions was more than four in as many as $18 \%$ of patients [29]. There is no conclusive evidence that multifocal IPMN pose a higher risk of HGD or invasive carcinoma, and we thus recommend that each cyst be assessed individually, rather than performing a total pancreatectomy upfront [10, 48, 72]. More critically, concomitant PDAC may develop in $3-11 \%$ of patients, either in the primary resection specimen or as a metachronous event [73-75]. While our experience suggests that metachronous PDAC after resection for IPMN is uncommon (0.5\%), other series have reported a 5 - and 10-year cumulative incidence of 4.5 and 5.9\%, respectively [76]. Hence, we also support continuing surveillance of surgically fit patients after resection of non-invasive IPMN [10].

The importance of achieving a clear resection margin to prevent disease recurrence is controversial. It is widely accepted that clear evidence of HGD or invasive carcinoma at the resection margin is a very strong predictor of disease recurrence and warrants further surgical resection $[10,69]$. The clinical relevance of LGD at the resection margins, however, is not well established. In our institutional experience, approximately $16 \%$ of patients had positive resection margins ( $71 \%$ in the form of LGD, $15 \%$ HGD, and $14 \%$ invasive carcinoma), and this group had a significantly higher risk of recurrence compared with patients with negative margins (25 vs. $14 \%, p=0.008$ ) [69]. Our findings are largely consistent with a study from Memorial Sloan Kettering Cancer Center [77], in which 
the presence of IPMN (LGD or HGD) or pancreatic intraepithelial neoplasia at the resection margins was significantly associated with disease recurrence; nevertheless, the vast majority of recurrences did not occur at the resection margins but elsewhere in the pancreatic remnant. Thus, if the rationale for extended resections with margins clear of LGD is to prevent recurrent disease at the resection margin, current evidence does not support it. To add to this issue, a study from the Johns Hopkins Hospital found no evidence that margin positivity (LGD or HGD) was associated with recurrence anywhere in the gland [78]. The discordance in the literature is likely a result of underpowered analyses assessing an incidence that occurs with a low frequency. Further, this entire discussion rests heavily on the premise that an expert pathologist is present to assess the margins during intraoperative consultation (frozen section); such assessment presents great challenges, including differentiating hyperplasia from neoplasia, and interpreting the significance of a margin that only shows denuded epithelium and inflammation, which may be an indication of an adjacent tumor [10].

\section{Follow-up of Non-Resected Patients}

The surveillance of patients with non-resected BD-IPMN is a contentious issue, which continues to spur debate among experts in the field. Advocates for continued surveillance base their arguments not only upon the potential for progression within IPMN but also upon the risk of developing concomitant PDAC elsewhere in the gland [79, 80]. Initial patient assessment should include investigation with dualphase pancreatic protocol CT and MRI or MRCP; whether to additionally perform EUS depends on the presence of symptoms and cyst characteristics. As we discussed previously, analysis of the different components of the cyst fluid remains a highly complex exercise and, if performed, it should ideally take place in centers with extensive experience caring for patients with this disease and its results should be interpreted with caution. The independent value of circulating markers (e.g. serum CA 19-9, blood NLR) remains to be confirmed, and the inclusion of elevated serum CA 19-9 as a worrisome feature in the revised IAP 2012 Fukuoka consensus guidelines will likely motivate validation studies of its utility.

There is no firm evidence to guide the periodicity or the duration of surveillance after deciding that a patient does not meet the criteria for resection. Retrospective studies have provided important clues on this issue, but ultimately, long-term prospective series will be necessary to answer this question more decisively. Current IAP guidelines use cyst size and growth as the main pacemakers for surveillance periodicity [10]. For cysts less than $2 \mathrm{~cm}$ in size, they recommend imaging with CT or MRI every 6 months, which may be spaced out to every 2 years if there are no changes. In patients with cysts $>2 \mathrm{~cm}$, more frequent evaluation with EUS (every 3-6 months) is recommended early on, followed by annual surveillance alternating EUS with MRI; these patients represent a tremendous challenge, especially young individuals with long life expectancy for whom the risk of pancreatic resection must be weighed against the financial and emotional cost of continued surveillance. The AGA guidelines [11] are much less granular regarding patient surveillance, recommending evaluation every 2 years regardless of the size of the cyst. Our surveillance strategy at MGH consists of evaluation every 6 months for the first year, then annually for the next 3 years if there is no evidence of cyst growth or development of new features (e.g. mural nodules, wall thickening), and then every 2 years.

The growing number of asymptomatic pancreatic cysts being diagnosed has placed a strong emphasis on the duration of followup of patients not undergoing surgery. The AGA guidelines recommend discontinuation of surveillance after 5 years of initial diagnosis if there are no significant changes in the cyst. Our experience demonstrates that this is not a safe strategy, with cases of invasive carcinoma arising from IPMN after 10 years of initial diagnosis $[17,81]$. In a series of 577 patients with suspected or presumed BD-IPMN under surveillance at MGH, we recently showed that risk of pancreatic malignancy (defined in this study as HGD or invasive carcinoma arising from IPMN, or concomitant PDAC) does not normalize after 5 years [17]. Among patients who were followed for more than 5 years, pancreatic malignancy developed in $5.5 \%$ as well as in $4.1 \%$ of those followed for more than 10 years. Notably, among patients who reached 5 years of follow-up without developing worrisome features, the age-standardized risk of pancreatic malignancy was 18.8 -fold (95\% CI 9.7-32.8; p < 0.001 ) higher compared to that of the US general population. Thus, we strongly advocate for continued surveillance of patients eligible for surgery after 5 years of the initial diagnosis. Last, this study also allowed us to identify a subgroup of patients in whom the risk of pancreatic malignancy is very low and who may be candidates for limited surveillance. We observed that among patients whose cysts remained equal or smaller than $1.5 \mathrm{~cm}$ throughout 5 years, only one case of pancreatic malignancy developed, and it was a concomitant PDAC away from the cyst.

\section{IAP and AGA Guidelines: How We Use Them}

The IAP consensus guidelines have evolved substantially since their first edition in 2006 [8]. The IAP Sendai consensus guidelines recommended surgical resection for i) all patients with MD-IPMN and ii) patients with BD-IPMN demonstrating either a) clinical symptoms, b) cysts $\geq 3 \mathrm{~cm}$ in size, or c) mural nodules. These guidelines proved to be very safe in terms of invasive carcinoma (most series reported that practically no cases were missed if these guidelines were followed), while missing approximately $6.5 \%$ of patients with HDG $[29,47]$. However, evidence suggesting that adherence to these guidelines resulted in overtreatment (patients undergoing surgery for BD-IPMN not showing HGD or invasive carcinoma [82]) led to the IAP 2012 Fukuoka consensus guidelines, which took a more conservative stance. The Fukuoka guidelines de-emphasized the importance of cyst size, which now is no longer an indication for resection by itself, and introduced two layers of risk stratification: worrisome features and high-risk stigmata [9]. Worrisome features include acute pancreatitis, size $>3 \mathrm{~cm}$, thickened/enhancing cyst walls, MPD size 5-9 mm, non-enhancing mural nodules, lymphad- 
enopathy, and abrupt change in MPD caliber with distal pancreatic atrophy. Patients with any of these characteristics should undergo EUS and obtain cytology, and if the latter is positive, resection is recommended; if negative or inconclusive, then close surveillance with CT/MRI or EUS is warranted. Presence of high-risk stigmata, namely obstructive jaundice, an enhancing mural nodule, and MPD $\geq 10 \mathrm{~mm}$, is an indication for resection in surgically fit patients. The utility and accuracy of these new guidelines have been validated [49, 83], but our experience suggests that they may be too conservative. In a series of 563 patients with BD-IPMN undergoing resection or under surveillance at $\mathrm{MGH}$, we found that removing the $3 \mathrm{~cm}$ size threshold doubled the number of missed cases with HGD and would have led to one missed case of invasive carcinoma [29]. As mentioned earlier, we do not know the natural history of lesions with HGD, but the stakes are too high when dealing with pancreatic cancer. Thus, while at MGH we have largely followed the different versions of the IAP consensus guidelines, we recommend that the IAP 2012 Fukuoka consensus guidelines (and its recent revision) be used cautiously, especially in young patients with long life expectancy. Expectedly, not all validation studies have agreed with the IAP consensus guidelines. Two studies - one from the University of Heidelberg and another from the H. Lee Moffitt Cancer Center - have reported that the combined risk of HGD or invasive carcinoma in BD-IPMN is $25-57 \%$ for sub-centimeter cysts and $26-28 \%$ for cysts $1-2 \mathrm{~cm}$ in size $[84,85]$. These figures are perplexing, especially if we keep in mind that some $10 \%$ of individuals older than 70 years have a sub-centimeter pancreatic cyst. Our own experience and reports by other groups lead us to believe that this seemingly high prevalence of HGD and invasive carcinoma arising from very small BD-IPMN could be explained by the presence of a small IPMN with LGD adjacent to a concomitant conventional PDAC, as the distinction between the two events is often challenging [10, 74, 75].

The AGA guidelines put forth in 2015 have marked differences from any edition of the IAP consensus guidelines and from our institutional posture [11]. Among their 10 recommendations, the main difference in terms of determining who should undergo surgery is that resection is recommended only for patients with two of three concerning features (namely, size $>3 \mathrm{~cm}$, a mural nodule, or
MPD dilation) and with evidence of malignancy after EUS; at the same time, the authors recognize that the sensitivity of EUS-guided cytological analysis is only $60 \%$, which means that $40 \%$ of patients with cancer will be missed if we applied these criteria. Another critical departure from our practice is the recommendation to stop patient surveillance after IPMN resection. This point came as a shock to many surgeons who have cared for patients with IPMN in whom an invasive recurrence occurs as many as 11 years after primary resection [69] and runs contrary to current evidence on the risk of recurrence of IPMN and of concomitant PDAC. Last, as discussed in the previous section, the recommendation to discontinue follow-up of patients with non-resected IPMN after 5 years in the absence of cyst changes is not supported by evidence and is the most critical point where our perspective is not aligned with the AGA guidelines.

\section{Conclusions}

In summary, IPMN represents a precursor to pancreatic cancer that offers a unique window of opportunity to intervene and impact the historically poor prognosis of the disease. However, overutilization of surgery may result in unnecessary morbidity and mortality and should be tailored to the individual patient's risk of developing cancer. In our opinion, the 2012 IAP Fukuoka consensus guidelines are safe with the caveat that they should be exercised with caution in younger patients. Radiographic surveillance should be continued after resection of worrisome lesions given the continued risk of recurrence or development of metachronous lesions. Future studies should focus on the development of fluid and serum biomarkers that can more accurately identify lesions with HGD in the preoperative setting, which will allow for better risk stratification of IPMN.

\section{Disclosure Statement}

The authors have no conflicts of interest to disclose.

\section{References}

1 Valsangkar NP, Morales-Oyarvide V, Thayer SP, Ferrone CR, Wargo JA, Warshaw AL, Fernandez-del Castillo C: 851 resected cystic tumors of the pancreas: a 33-year experience at the Massachusetts General Hospital. Surgery 2012;152:S4-12.

2 Basturk O, Hong SM, Wood LD, et al.; Baltimore Consensus Meeting: A revised classification system and recommendations from the Baltimore consensus meeting for neoplastic precursor lesions in the pancreas. Am J Surg Pathol 2015;39:1730-1741.

3 Mino-Kenudson M, Fernandez-del Castillo C, Baba Y, Valsangkar NP, Liss AS, Hsu M, Correa-Gallego C, Ingkakul T, Perez Johnston R, Turner BG, Androutsopoulos V, Deshpande V, McGrath D, Sahani DV, Brugge WR, Ogino S, Pitman MB, Warshaw AL, Thayer SP: Prognosis of invasive intraductal papillary mucinous neoplasm depends on histological and precursor epithelial subtypes. Gut 2011;60:1712-1720.
4 Furukawa T, Hatori T, Fujita I, Yamamoto M, Kobayashi M, Ohike N, Morohoshi T, Egawa S, Unno M, Takao S, Osako M, Yonezawa S, Mino-Kenudson M, Lauwers GY, Yamaguchi H, Ban S, Shimizu M: Prognostic relevance of morphological types of intraductal papillary mucinous neoplasms of the pancreas. Gut 2011;60:509-516.

5 Wu J, Matthaei H, Maitra A, Dal Molin M, Wood LD, Eshleman JR, Goggins M, Canto MI, Schulick RD, Edil $\mathrm{BH}$, Wolfgang CL, Klein AP, Diaz LA Jr, Allen PJ, Schmidt CM, Kinzler KW, Papadopoulos N, Hruban RH, Vogelstein B: Recurrent GNAS mutations define an unexpected pathway for pancreatic cyst development. Sci Transl Med 2011;3:92ra66.

6 Furukawa T, Kuboki Y, Tanji E, Yoshida S, Hatori T, Yamamoto M, Shibata N, Shimizu K, Kamatani N, Shiratori K: Whole-exome sequencing uncovers frequent
GNAS mutations in intraductal papillary mucinous neoplasms of the pancreas. Sci Rep 2011;1:161.

7 Tamura K, Ohtsuka T, Date K, Fujimoto T, Matsunaga T, Kimura H, Watanabe Y, Miyazaki T, Ohuchida K, Takahata S, Ishigami K, Oda Y, Mizumoto K, Nakamura M, Tanaka M: Distinction of invasive carcinoma derived from intraductal papillary mucinous neoplasms from concomitant ductal adenocarcinoma of the pancreas using molecular biomarkers. Pancreas 2016;45:826-835.

8 Tanaka M, Chari S, Adsay V, Fernandez-del Castillo C, Falconi M, Shimizu M, Yamaguchi K, Yamao K, Matsuno S; International Association of Pancreatology: International consensus guidelines for management of intraductal papillary mucinous neoplasms and mucinous cystic neoplasms of the pancreas. Pancreatology 2006;6:17-32. 
9 Tanaka M, Fernandez-del Castillo C, Adsay V, Chari S Falconi M, Jang JY, Kimura W, Levy P, Pitman MB, Schmidt CM, Shimizu M, Wolfgang CL, Yamaguchi K, Yamao K; International Association of Pancreatology: International consensus guidelines 2012 for the management of IPMN and MCN of the pancreas. Pancreatology 2012;12:183-197.

10 Tanaka M, Fernandez-del Castillo C, Kamisawa T, Jang JY, Levy P, Ohtsuka T, Salvia R, Shimizu Y, Tada $\mathrm{M}$, Wolfgang CL: Revisions of international consensus Fukuoka guidelines for the management of IPMN of the pancreas. Pancreatology 2017;17:738-753.

11 Vege SS, Ziring B, Jain R, Moayyedi P; Clinical Guidelines Committee; American Gastroenterology Association: American Gastroenterological Association Institute guideline on the diagnosis and management of asymptomatic neoplastic pancreatic cysts. Gastroenterology 2015;148:819-822.

12 Scheiman JM, Hwang JH, Moayyedi P: American Gastroenterological Association technical review on the diagnosis and management of asymptomatic neoplastic pancreatic cysts. Gastroenterology 2015;148:824848.e822.

13 Laffan TA, Horton KM, Klein AP, Berlanstein B, Siegelman SS, Kawamoto S, Johnson PT, Fishman EK, Hruban RH: Prevalence of unsuspected pancreatic cysts on MDCT. AJR Am J Roentgenol 2008;191:802-807.

14 Zhang XM, Mitchell DG, Dohke M, Holland GA, Parker L: Pancreatic cysts: depiction on single-shot fast spin-echo MR images. Radiology 2002;223:547-553.

15 Fernandez-del Castillo C, Targarona J, Thayer SP, Rattner DW, Brugge WR, Warshaw AL: Incidental pancreatic cysts: clinicopathologic characteristics and comparison with symptomatic patients. Arch Surg 2003;138:427-433; discussion 433-434.

16 de Jong K, Nio CY, Hermans JJ, Dijkgraaf MG, Gouma DJ, van Eijck CH, van Heel E, Klass G, Fockens P, Bruno MJ: High prevalence of pancreatic cysts detected by screening magnetic resonance imaging examinations. Clin Gastroenterol Hepatol 2010;8:806-811.

17 Pergolini I, Sahora K, Ferrone CR, Morales-Oyarvide V, Wolpin BM, Mucci LA, Brugge WR, Mino-Kenudson M, Patino M, Sahani DV, Warshaw AL, Lillemoe KD, Fernandez-del Castillo C: Long-term risk of pancreatic malignancy in patients with branch duct intraductal papillary mucinous neoplasm in a referral center. Gastroenterology 2017;153:1284-1294.e1.

18 Waters JA, Schmidt CM, Pinchot JW, White PB, Cummings OW, Pitt HA, Sandrasegaran K, Akisik F, Howard TJ, Nakeeb A, Zyromski NJ, Lillemoe KD: CT vs MRCP: optimal classification of IPMN type and extent. J Gastrointest Surg 2008;12:101-109.

19 Baiocchi GL, Portolani N, Missale G, Baronchelli C, Gheza F, Cantu M, Grazioli L, Giulini SM: Intraductal papillary mucinous neoplasm of the pancreas (IPMN): clinico-pathological correlations and surgical indications. World J Surg Oncol 2010;8:25.

20 Sahora K, Fernandez-del Castillo C, Dong F, Marchegiani G, Thayer SP, Ferrone CR, Sahani DV, Brugge WR, Warshaw AL, Lillemoe KD, Mino-Kenudson M: Not all mixed-type intraductal papillary mucinous neoplasms behave like main-duct lesions: implications of minimal involvement of the main pancreatic duct. Surgery 2014;156:611-621.

21 Morales-Oyarvide V, Pergolini I, Ferrone CR, MinoKenudson M, Warshaw AL, Lillemoe KD, Fernandezdel Castillo C: Main pancreatic duct size independently predicts histological main duct involvement, intestinal phenotype, and malignancy in intraductal papillary mucinous neoplasm. Pancreas 2016;45:1526.

22 Adsay NV, Kloppel G, Fukushima N: Intraductal neoplasms of the pancreas; in Bosman FT, Carneiro F, Hruban RH, Theise ND (eds): WHO Classification of Tumors. Lyon, WHO Press, 2010, pp 304-313.
23 Adsay V, Mino-Kenudson M, Furukawa T, et al.; Members of Verona Consensus Meeting, 2013: Pathologic evaluation and reporting of intraductal papillary mucinous neoplasms of the pancreas and other tumoral intraepithelial neoplasms of pancreatobiliary tract: recommendations of Verona consensus meeting. Ann Surg 2016;263:162-177.

24 Adsay NV, Merati K, Basturk O, Iacobuzio-Donahue C, Levi E, Cheng JD, Sarkar FH, Hruban RH, Klimstra DS: Pathologically and biologically distinct types of epithelium in intraductal papillary mucinous neoplasms: delineation of an 'intestinal' pathway of carcinogenesis in the pancreas. Am J Surg Pathol 2004;28: 839-848.

25 Mohri D, Asaoka Y, Ijichi H, Miyabayashi K, Kudo Y, Seto M, Ohta M, Tada M, Tanaka Y, Ikenoue T, Tateishi K, Isayama H, Kanai F, Fukushima N, Tada M, Kawabe T, Omata M, Koike K: Different subtypes of intraductal papillary mucinous neoplasm in the pancreas have distinct pathways to pancreatic cancer progression. J Gastroenterol 2012;47:203-213.

26 Tan MC, Basturk O, Brannon AR, Bhanot U, Scott SN, Bouvier N, LaFemina J, Jarnagin WR, Berger MF, Klimstra D, Allen PJ: GNAS and KRAS mutations define separate progression pathways in intraductal papillary mucinous neoplasm-associated carcinoma. J Am Coll Surg 2015;220:845-854.e841.

27 Marchegiani G, Mino-Kenudson M, Ferrone CR, Warshaw AL, Lillemoe KD, Fernandez-del Castillo C: Oncocytic-type intraductal papillary mucinous neoplasms: a unique malignant pancreatic tumor with good long-term prognosis. J Am Coll Surg 2015;220:839-844.

28 Morales-Oyarvide V, Mino-Kenudson M, Ferrone C, Warshaw AL, Lillemoe KD, Sahani DV, Pergolini I, Attiyeh M, Al Efishat M, Rezaee N, Hruban RH, He J, Weiss MJ, Allen PJ, Wolfgang CL, Fernandez-del Castillo C: Intraductal papillary mucinous neoplasm of the pancreas in young patients: tumor biology, clinical features, and survival outcomes. J Gastrointest Surg 2017;DOI: 10.1007/s11605-017-3602-z.

29 Sahora K, Mino-Kenudson M, Brugge W, Thayer SP, Ferrone CR, Sahani D, Pitman MB, Warshaw AL, Lillemoe KD, Fernandez-del Castillo C: Branch duct intraductal papillary mucinous neoplasms: does cyst size change the tip of the scale? A critical analysis of the revised international consensus guidelines in a large single-institutional series. Ann Surg 2013;258:466-475.

30 Pannala R, Leirness JB, Bamlet WR, Basu A, Petersen GM, Chari ST: Prevalence and clinical profile of pancreatic cancer-associated diabetes mellitus. Gastroenterology 2008;134:981-987.

31 Morales-Oyarvide V, Mino-Kenudson M, Ferrone CR, Sahani DV, Pergolini I, Negreros-Osuna AA, Warshaw AL, Lillemoe KD, Fernandez-del Castillo C: Diabetes mellitus in intraductal papillary mucinous neoplasm of the pancreas is associated with high-grade dysplasia and invasive carcinoma. Pancreatology 2017;17:920-926.

32 Morales-Oyarvide V, Mino-Kenudson M, Ferrone CR, Gonzalez-Gonzalez LA, Warshaw AL, Lillemoe KD, Fernandez-del Castillo C: Acute pancreatitis in intraductal papillary mucinous neoplasms: a common predictor of malignant intestinal subtype. Surgery 2015; 158:1219-1225

33 Ferrone CR, Finkelstein DM, Thayer SP, Muzikansky A, Fernandez-del Castillo C, Warshaw AL: Perioperative CA19-9 levels can predict stage and survival in patients with resectable pancreatic adenocarcinoma. J Clin Oncol 2006;24:2897-2902.

34 Wang W, Zhang L, Chen L, Wei J, Sun Q, Xie Q, Zhou X, Zhou D, Huang P, Yang Q, Xie H, Zhou L, Zheng S: Serum carcinoembryonic antigen and carbohydrate antigen 19-9 for prediction of malignancy and invasiveness in intraductal papillary mucinous neoplasms of the pancreas: a meta-analysis. Biomed Rep 2015;3:43-50.
35 Morales-Oyarvide V, Mino-Kenudson M, Ferrone CR, Warshaw AL, Lillemoe KD, Fernandez-del Castillo C: Sul353 elevated serum CA19-9 in IPMN is a highlyspecific marker of invasive cancer and an independent predictor of advanced stage and poor survival. Gastroenterology 2016;150:S501.

36 Kim JR, Jang JY, Kang MJ, Park T, Lee SY, Jung W, Chang J, Shin Y, Han Y, Kim SW: Clinical implication of serum carcinoembryonic antigen and carbohydrate antigen 19-9 for the prediction of malignancy in intraductal papillary mucinous neoplasm of pancreas. J Hepatobiliary Pancreat Sci 2015;22:699-707.

37 Fritz S, Hackert T, Hinz U, Hartwig W, Buchler MW Werner J: Role of serum carbohydrate antigen 19-9 and carcinoembryonic antigen in distinguishing between benign and invasive intraductal papillary mucinous neoplasm of the pancreas. Br J Surg 2011;98:104-110.

38 Arima K, Okabe H, Hashimoto D, Chikamoto A, Kuroki H, Taki K, Kaida T, Higashi T, Nitta H, Komohara Y, Beppu T, Takeya M, Baba H: The neutrophilto-lymphocyte ratio predicts malignant potential in intraductal papillary mucinous neoplasms. J Gastrointest Surg 2015;19:2171-2177.

39 Gemenetzis G, Bagante F, Griffin JF, Rezaee N, Javed AA, Manos LL, Lennon AM, Wood LD, Hruban RH, Zheng L, Zaheer A, Fishman EK, Ahuja N, Cameron JL, Weiss MJ, He J, Wolfgang CL: Neutrophil-to-lymphocyte ratio is a predictive marker for invasive malignancy in intraductal papillary mucinous neoplasms of the pancreas. Ann Surg 2017;266:339-345.

40 Sainani NI, Saokar A, Deshpande V, Fernandez-del Castillo C, Hahn P, Sahani DV: Comparative performance of MDCT and MRI with MR cholangiopancreatography in characterizing small pancreatic cysts. AJR Am J Roentgenol 2009;193:722-731.

41 Sahani DV, Sainani NI, Blake MA, Crippa S, MinoKenudson M, Fernandez-del Castillo C: Prospective evaluation of reader performance on MDCT in characterization of cystic pancreatic lesions and prediction of cyst biologic aggressiveness. AJR Am J Roentgenol 2011;197:W53-61.

42 Hirono S, Tani M, Kawai M, Okada K, Miyazawa M, Shimizu A, Kitahata Y, Yamaue H: The carcinoembryonic antigen level in pancreatic juice and mural nodule size are predictors of malignancy for branch duct type intraductal papillary mucinous neoplasms of the pancreas. Ann Surg 2012;255:517-522.

43 Ohno E, Itoh A, Kawashima H, Ishikawa T, Matsubara H, Itoh Y, Nakamura Y, Hiramatsu T, Nakamura M, Miyahara R, Ohmiya N, Ishigami M, Katano Y, Goto $\mathrm{H}$, Hirooka Y: Malignant transformation of branch duct-type intraductal papillary mucinous neoplasms of the pancreas based on contrast-enhanced endoscopic ultrasonography morphological changes: focus on malignant transformation of intraductal papillary mucinous neoplasm itself. Pancreas 2012;41:855-862.

44 Seo N, Byun JH, Kim JH, Kim HJ, Lee SS, Song KB, Kim SC, Han DJ, Hong SM, Lee MG: Validation of the 2012 international consensus guidelines using computed tomography and magnetic resonance imaging: branch duct and main duct intraductal papillary mucinous neoplasms of the pancreas. Ann Surg 2016;263:557-564.

45 Berland LL, Silverman SG, Gore RM, Mayo-Smith WW, Megibow AJ, Yee J, Brink JA, Baker ME, Federle MP, Foley WD, Francis IR, Herts BR, Israel GM, Krinsky G, Platt JF, Shuman WP, Taylor AJ: Managing incidental findings on abdominal CT: white paper of the ACR incidental findings committee. J Am Coll Radiol 2010;7:754-773.

46 Salvia R, Fernandez-del Castillo C, Bassi C, Thayer SP, Falconi M, Mantovani W, Pederzoli P, Warshaw AL: Main-duct intraductal papillary mucinous neoplasms of the pancreas: clinical predictors of malignancy and long-term survival following resection. Ann Surg 2004; 239:678-685; discussion 685-687. 
47 Fernandez-del Castillo C, Adsay NV: Intraductal papillary mucinous neoplasms of the pancreas. Gastroenterology 2010;139:708-713.

48 Rodriguez JR, Salvia R, Crippa S, Warshaw AL, Bassi C, Falconi M, Thayer SP, Lauwers GY, Capelli P, Mino-Kenudson M, Razo O, McGrath D, Pederzoli P, Fernandez-del Castillo C: Branch-duct intraductal papillary mucinous neoplasms: observations in $145 \mathrm{pa}-$ tients who underwent resection. Gastroenterology 2007;133:72-79.

49 Kim KW, Park SH, Pyo J, Yoon SH, Byun JH, Lee MG Krajewski KM, Ramaiya NH: Imaging features to distinguish malignant and benign branch-duct type intraductal papillary mucinous neoplasms of the pancreas: a meta-analysis. Ann Surg 2014;259:72-81.

50 Anand N, Sampath K, Wu BU: Cyst features and risk of malignancy in intraductal papillary mucinous neoplasms of the pancreas: a meta-analysis. Clin Gastroenterol Hepatol 2013;11:913-921.

51 Kitano M, Sakamoto H, Komaki T, Kudo M: New techniques and future perspective of EUS for the differential diagnosis of pancreatic malignancies: contrast harmonic imaging. Dig Endosc 2011;23(suppl 1):46-50

52 Correa-Gallego C, Warshaw AL, Fernandez-del Castillo C: Fluid CEA in IPMNs: a useful test or the flip of a coin? Am J Gastroenterol 2009;104:796-797.

53 Brugge WR, Lewandrowski K, Lee-Lewandrowski E, Centeno BA, Szydlo T, Regan S, Fernandez-del Castillo C, Warshaw AL: Diagnosis of pancreatic cystic neoplasms: a report of the cooperative pancreatic cyst study. Gastroenterology 2004;126:1330-1336.

54 Cizginer S, Turner BG, Bilge AR, Karaca C, Pitman $\mathrm{MB}$, Brugge WR: Cyst fluid carcinoembryonic antigen is an accurate diagnostic marker of pancreatic mucinous cysts. Pancreas 2011;40:1024-1028.

55 Das KK, Xiao H, Geng X, Fernandez-del Castillo C, Morales-Oyarvide V, Daglilar E, Forcione DG, Bounds BC, Brugge WR, Pitman MB, Mino-Kenudson M, Das KM: $\mathrm{mAb}$ Das-1 is specific for high-risk and malignant intraductal papillary mucinous neoplasm (IPMN). Gut 2014;63:1626-1634.

56 Das KK, Geng XG, Morales-Oyarvide V, Huynh T, Pergolini I, Pitman MB, Ferrone C, Brugge W, Al Efishat M, Haviland D, Thompson E, Wolfgang C, Lennon AM, Allen P, Lillemoe KD, Fernandez-del Castillo C, Das KM, Mino-Kenudson M: 666 - A multicenter, validation study of cyst fluid analysis for $\mathrm{mAb}$-Das1 for the identification of high-risk and malignant mucinous cysts of the pancreas. Gastroenterology 2017;152:S146-S147.

57 Pitman MB, Genevay M, Yaeger K, Chebib I, Turner BG, Mino-Kenudson M, Brugge WR: High-grade atypical epithelial cells in pancreatic mucinous cysts are a more accurate predictor of malignancy than 'positive' cytology. Cancer Cytopathol 2010;118:434-440.

58 Pitman MB, Yaeger KA, Brugge WR, Mino-Kenudson M: Prospective analysis of atypical epithelial cells as a high-risk cytologic feature for malignancy in pancreatic cysts. Cancer Cytopathol 2013;121:29-36.

59 Fritz S, Fernandez-del Castillo C, Mino-Kenudson M, Crippa S, Deshpande V, Lauwers GY, Warshaw AL, Thayer SP, Iafrate AJ: Global genomic analysis of intraductal papillary mucinous neoplasms of the pancreas reveals significant molecular differences compared to ductal adenocarcinoma. Ann Surg 2009;249:440-447.

60 Jones M, Zheng Z, Wang J, Dudley J, Albanese E, Kadayifci A, Dias-Santagata D, Le L, Brugge WR, Fernandez-del Castillo C, Mino-Kenudson M, Iafrate AJ, Pitman MB: Impact of next-generation sequencing on the clinical diagnosis of pancreatic cysts. Gastrointest Endosc 2016;83:140-148.
61 Springer S, Wang Y, Dal Molin M, et al: A combination of molecular markers and clinical features improve the classification of pancreatic cysts. Gastroenterology 2015;149:1501-1510.

62 Crippa S, Fernandez-del Castillo C, Salvia R, Finkelstein D, Bassi C, Dominguez I, Muzikansky A, Thayer SP, Falconi M, Mino-Kenudson M, Capelli P, Lauwers GY, Partelli S, Pederzoli P, Warshaw AL: Mucin-producing neoplasms of the pancreas: an analysis of distinguishing clinical and epidemiologic characteristics. Clin Gastroenterol Hepatol 2010;8:213-219.

63 Fernandez-del Castillo C, Morales-Oyarvide V, McGrath D, Wargo JA, Ferrone CR, Thayer SP, Lillemoe KD, Warshaw AL: Evolution of the Whipple procedure at the Massachusetts General Hospital. Surgery 2012;152:S56-63.

64 Fong ZV, Alvino DM, Fernandez-del Castillo C, Nipp RD, Traeger LN, Ruddy M, Lubitz CC, Johnson CD, Chang DC, Warshaw AL, Lillemoe KD, Ferrone CR: Health-related quality of life and functional outcomes in 5-year survivors after pancreaticoduodenectomy. Ann Surg 2017;266:685-692.

65 Sahora K, Ferrone CR, Brugge WR, Morales-Oyarvide V, Warshaw AL, Lillemoe KD, Fernandez-del Castillo C: Effects of comorbidities on outcomes of patients with intraductal papillary mucinous neoplasms. Clin Gastroenterol Hepatol 2015;13:1816-1823.

66 Sauvanet A, Gaujoux S, Blanc B, Couvelard A, Dokmak S, Vullierme MP, Ruszniewski P, Belghiti J, Levy P: Parenchyma-sparing pancreatectomy for presumed noninvasive intraductal papillary mucinous neoplasms of the pancreas. Ann Surg 2014;260:364-371.

67 Faitot F, Gaujoux S, Barbier L, Novaes M, Dokmak S, Aussilhou B, Couvelard A, Rebours V, Ruszniewski P, Belghiti J, Sauvanet A: Reappraisal of pancreatic enucleations: a single-center experience of 126 procedures. Surgery 2015;158:201-210.

68 Kaiser J, Fritz S, Klauss M, Bergmann F, Hinz U, Strobel O, Schneider L, Buchler MW, Hackert T: Enucleation: a treatment alternative for branch duct intraductal papillary mucinous neoplasms. Surgery 2017; 161:602-610.

69 Marchegiani G, Mino-Kenudson M, Ferrone CR, Morales-Oyarvide V, Warshaw AL, Lillemoe KD, Fernandez-del Castillo C: Patterns of recurrence after resection of IPMN: who, when, and how? Ann Surg 2015; 262:1108-1114.

70 Kang MJ, Jang JY, Lee KB, Chang YR, Kwon W, Kim SW: Long-term prospective cohort study of patients undergoing pancreatectomy for intraductal papillary mucinous neoplasm of the pancreas: implications for postoperative surveillance. Ann Surg 2014;260:356-363.

71 White R, D'Angelica M, Katabi N, Tang L, Klimstra D, Fong Y, Brennan M, Allen P: Fate of the remnant pancreas after resection of noninvasive intraductal papillary mucinous neoplasm. J Am Coll Surg 2007;204: 987-993; discussion 993-995.

72 Salvia R, Partelli S, Crippa S, Landoni L, Capelli P, Manfredi R, Bassi C, Pederzoli P: Intraductal papillary mucinous neoplasms of the pancreas with multifocal involvement of branch ducts. Am J Surg 2009;198:709-714.

73 Marchegiani G, Mino-Kenudson M, Sahora K, Morales-Oyarvide V, Thayer S, Ferrone $\mathrm{C}$, Warshaw AL, Lillemoe KD, Fernandez-del Castillo C: IPMN involving the main pancreatic duct: biology, epidemiology, and long-term outcomes following resection. Ann Surg 2015;261:976-983.

74 Ingkakul T, Sadakari Y, Ienaga J, Satoh N, Takahata S, Tanaka M: Predictors of the presence of concomitant invasive ductal carcinoma in intraductal papillary mucinous neoplasm of the pancreas. Ann Surg 2010;251: 70-75.
75 Ideno N, Ohtsuka T, Kono H, Fujiwara K, Oda Y, Aishima S, Ito T, Ishigami K, Tokunaga S, Ohuchida K, Takahata S, Nakamura M, Mizumoto K, Tanaka M: Intraductal papillary mucinous neoplasms of the pancreas with distinct pancreatic ductal adenocarcinomas are frequently of gastric subtype. Ann Surg 2013;258: 141-151.

76 Miyasaka Y, Ohtsuka T, Tamura K, Mori Y, Shindo K, Yamada D, Takahata S, Ishigami K, Ito T, Tokunaga S, Oda Y, Mizumoto K, Nakamura M, Tanaka M: Predictive factors for the metachronous development of high-risk lesions in the remnant pancreas after partial pancreatectomy for intraductal papillary mucinous neoplasm. Ann Surg 2016;263:1180-1187.

77 Frankel TL, LaFemina J, Bamboat ZM, D’Angelica MI, DeMatteo RP, Fong Y, Kingham TP, Jarnagin WR, Allen PJ: Dysplasia at the surgical margin is associated with recurrence after resection of non-invasive intraductal papillary mucinous neoplasms. HPB (Oxford) 2013;15:814-821.

78 He J, Cameron JL, Ahuja N, Makary MA, Hirose K, Choti MA, Schulick RD, Hruban RH, Pawlik TM, Wolfgang CL: Is it necessary to follow patients after resection of a benign pancreatic intraductal papillary mucinous neoplasm? J Am Coll Surg 2013;216:657-665.

79 Ohtsuka T, Kono H, Tanabe R, Nagayoshi Y, Mori Y, Sadakari Y, Takahata S, Oda Y, Aishima S, Igarashi $\mathrm{H}$, Ito T, Ishigami K, Nakamura M, Mizumoto K, Tanaka M: Follow-up study after resection of intraductal papillary mucinous neoplasm of the pancreas; special references to the multifocal lesions and development of ductal carcinoma in the remnant pancreas. Am J Surg 2012;204:44-48.

80 Tanno S, Nakano Y, Sugiyama Y, Nakamura K, Sasajima J, Koizumi K, Yamazaki M, Nishikawa T, Mizukami Y, Yanagawa N, Fujii T, Obara T, Okumura T, Kohgo Y: Incidence of synchronous and metachronous pancreatic carcinoma in 168 patients with branch duct intraductal papillary mucinous neoplasm. Pancreatology 2010;10:173-178.

81 Honselmann KC, Patino M, Mino-Kenudson M, Ferrone C, Warshaw AL, Fernandez-del Castillo C, Lillemoe KD: Ductal carcinoma arising in a largely unchanged presumed branch-duct IPMN after 10 years of surveillance. Ann Surg 2017;266:e38-e40.

82 Pelaez-Luna M, Chari ST, Smyrk TC, Takahashi N, Clain JE, Levy MJ, Pearson RK, Petersen BT, Topazian MD, Vege SS, Kendrick M, Farnell MB: Do consensus indications for resection in branch duct intraductal papillary mucinous neoplasm predict malignancy? A study of 147 patients. Am J Gastroenterol 2007;102: 1759-1764.

83 Goh BK, Tan DM, Thng CH, Lee SY, Low AS, Chan CY, Wong JS, Lee VT, Cheow PC, Chow PK, Chung AY, Wong WK, Ooi LL: Are the Sendai and Fukuoka consensus guidelines for cystic mucinous neoplasms of the pancreas useful in the initial triage of all suspected pancreatic cystic neoplasms? A single-institution experience with 317 surgically-treated patients. Ann Surg Oncol 2014;21:1919-1926.

84 Fritz S, Klauss M, Bergmann F, Hackert T, Hartwig W, Strobel O, Bundy BD, Buchler MW, Werner J: Small (Sendai negative) branch-duct IPMNs: not harmless. Ann Surg 2012;256:313-320.

85 Wong J, Weber J, Centeno BA, Vignesh S, Harris CL, Klapman JB, Hodul P: High-grade dysplasia and adenocarcinoma are frequent in side-branch intraductal papillary mucinous neoplasm measuring less than 3 $\mathrm{cm}$ on endoscopic ultrasound. J Gastrointest Surg 2013;17:78-84; discussion 84-85. 